\title{
Just the facts: point-of-care ultrasound for painless loss of vision
}

\author{
Michael Gottlieb ${ }^{1}$ (D) Amy E. Marks ${ }^{1} \cdot$ Nathan $\mathrm{Hecht}^{2} \cdot$ Paul Atkinson $^{3}$
}

Received: 12 March 2021 / Accepted: 17 June 2021 / Published online: 29 June 2021

(C) The Author(s), under exclusive licence to Canadian Association of Emergency Physicians (CAEP)/ Association Canadienne de Médecine d'Urgence (ACMU) 2021

\section{Clinical scenario}

A 42-year-old woman presents with new painless loss of vision in her left eye. The symptoms have been present since yesterday and began with blurred vision, progressing to loss of vision on the temporal aspect of her left eye. Her external ocular examination including slit lamp examination is unremarkable and her intraocular pressures are $18 \mathrm{mmHg}$ bilaterally. In-person ophthalmology consultation is not available in the Emergency Department and the clinician wonders whether this could be a retinal detachment.

\section{Key clinical questions}

\section{How do you perform ocular ultrasound?}

Ocular point-of-care ultrasound (POCUS) is best performed in a supine or recumbent position. A high-frequency linear probe of $7.5 \mathrm{MHz}$ or higher is ideal and should be used with specific ocular presets to reduce the potential risk of thermal injury to the eye [1]. Copious gel should be applied to the closed eyelid to act as an acoustic coupler and spacer.

Michael Gottlieb

michaelgottliebmd@gmail.com

Amy E. Marks

amy.e.marks@gmail.com

Nathan Hecht

nhecht@toh.ca

Paul Atkinson

paul.atkinson@dal.ca

1 Department of Emergency Medicine, Rush University Medical Center, 1750 West Harrison Street, Suite 108 Kellogg, Chicago, IL 60612, USA

2 Department of Emergency Medicine, The Ottawa Hospital, University of Ottawa, Ottawa, ON, Canada

3 Department of Emergency Medicine, Dalhousie University, Horizon Health, Saint John, NB, Canada
A barrier (e.g., Tegaderm ${ }^{\mathrm{TM}}$ ) is optional to prevent getting gel in the eye, but care must be made to avoid air getting trapped between the barrier and the eyelid as this can limit the POCUS image quality. Bracing the probe-hand on the face (e.g., maxilla or nasal bridge) will help prevent excess pressure on the eye [2]. The orbit should be scanned in both the transverse and longitudinal planes (Fig. 1A). To assess the lateral walls, the globe should be visualized with the patient looking straight ahead, followed by in both extremes (i.e., left and right in transverse, superior and inferior in sagittal). Once the retina has been visualized, ask the patient to move their eyes from side to side to assist in characterizing pathologies (e.g., a tethered retinal detachment does not move as freely as a posterior vitreous detachment) [3].

\section{How accurate is the history and physical examination for retinal detachment and tears?}

While clear indications for referral are monocular vision loss or a visual field deficit, data on other findings in retinal detachments are more limited [4]. Studies of retinal tears, which are initial breaks in the retina that can lead to more significant detachment, have reported variable diagnostic accuracy for the history and physical examination. Subjective vision loss has only a moderate impact on the diagnosis with a positive likelihood ratio $(\mathrm{LR}+)$ of 5.0 and a negative likelihood ratio (LR-) of 0.60 [4]. Similarly, flashes and floaters have a combined LR + of 1.2 and a LR- of 0.90 [4]. Vitreous pigment has a much greater diagnostic role with a LR + of 44 and LR - of 0.23 but can be more challenging to identify and is not typically assessed by Emergency Medicine clinicians [4].

\section{What is the accuracy of ultrasound for diagnosing retinal detachment and vitreous hemorrhage?}

A meta-analysis of 11 studies found that POCUS was $94 \%$ sensitive and $96 \%$ specific for retinal detachments [5]. For vitreous hemorrhage, a different systematic review reported 
Fig. 1 A Normal eye. The star identifies the anterior chamber, the arrowhead identifies the lens, and the optic nerve sheath diameter is measured using the cross-hairs with the letter B. Image courtesy of Dr. Mandy Peach. B Retinal detachment. The large arrow identifies the retinal detachment. C Vitreous detachment. The small arrows identify the vitreous detachment. Image courtesy of Dr. Mandy Peach

that POCUS was $90 \%$ sensitive and $92 \%$ specific, while for vitreous detachment POCUS was $67 \%$ sensitive and $89 \%$ specific [6]. A subsequent prospective multicenter study reported that POCUS was $97 \%$ sensitive and $88 \%$ specific for retinal detachments, $82 \%$ sensitive and $82 \%$ specific for vitreous hemorrhage, and $43 \%$ sensitive and $96 \%$ specific for vitreous detachment. [7]. When evaluating the posterior retina, it is important to distinguish retinal detachment from posterior vitreous detachment (Fig. 1B, C) [8]. Table 1 highlights features used to distinguish retinal detachment from posterior vitreous detachment. Importantly, POCUS has poor sensitivity for retinal tears, with $42 \%$ sensitivity and $82 \%$ specificity [9]. Therefore, the absence of retinal detachment on POCUS still warrants close outpatient follow-up. Readers should also note that the differential diagnosis of painless vision loss can be expansive and there are several causes which would not be readily identified with ultrasound (e.g., retinal vein occlusion, non-central retinal artery occlusions, metabolic and toxicologic causes, intracerebral etiologies). Therefore, it is important to combine the use of POCUS with a thorough history and examination.

\section{What is the role for ultrasound in diagnosing lens detachments and ocular foreign bodies?}

POCUS is a useful adjunct to diagnose lens dislocation. One systematic review found that POCUS had $97 \%$ sensitivity and $99 \%$ specificity for identifying a lens dislocation [6]. On ultrasound, a lens dislocation will appear as an object with hyperechoic walls and an anechoic center floating in the posterior chamber of the eye. With kinetic movement of the eye, the object will move within the posterior chamber. POCUS may also be used to identify ocular foreign bodies, with a sensitivity of $100 \%$ and specificity of $99 \%$ [6]. An ocular foreign body will appear as a hyperechoic structure in the posterior or anterior chamber of the eye. Depending on the material, ocular foreign bodies may have posterior shadowing or reverberation artifacts. It is important to note that the literature is more limited for these applications and is based primarily on two studies comprising 400 total patients [6].

\section{What is the role for ultrasound in diagnosing increased intracranial pressure?}

Increased intracranial pressure can present with blurred vision, which is more common bilaterally. Two components
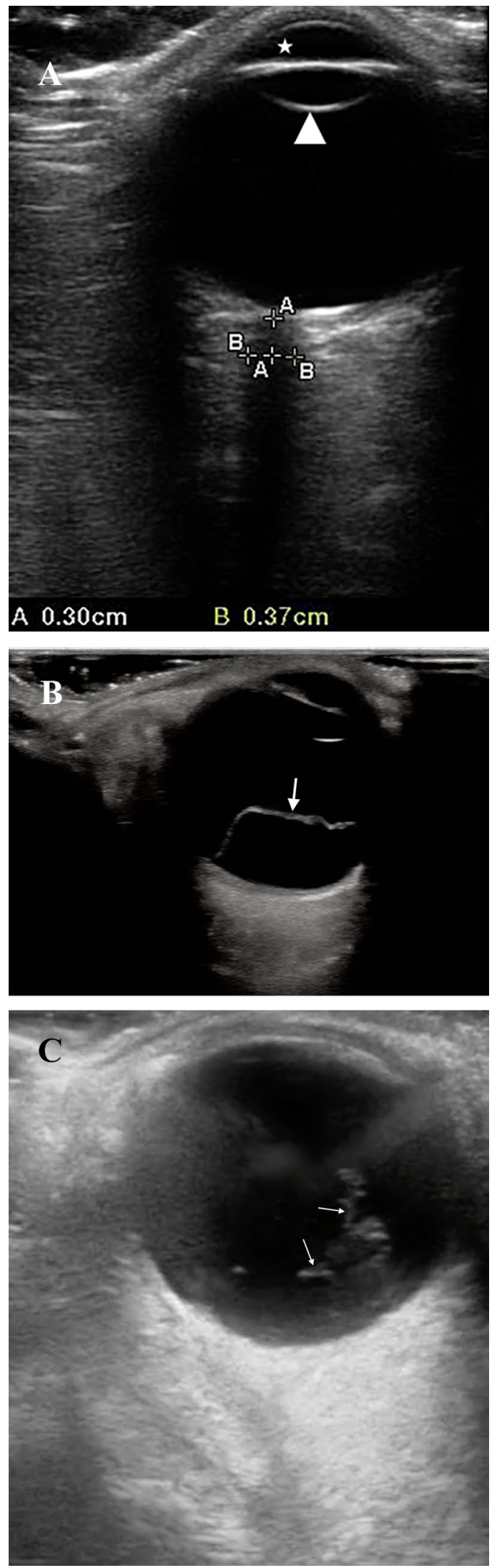
Table 1 Comparison of retinal detachment with posterior vitreous detachment on ultrasound

\begin{tabular}{ll}
\hline Retinal detachments & Posterior vitreous detachments \\
\hline $\begin{array}{l}\text { Does not cross the optic nerve } \\
\text { Uniformly thick }\end{array}$ & $\begin{array}{l}\text { Can cross the optic nerve } \\
\text { Varying degrees of thickness and often thinner than } \\
\text { retinal detachment }\end{array}$ \\
More echogenic & Less echogenic \\
Less mobile with ocular movements & More mobile with ocular movements \\
May be tethered directly to the optic nerve & Can be focal or extensive \\
Become thicker and less mobile over time & Often present with hemorrhage or inflammatory debris \\
\hline
\end{tabular}

of ocular POCUS can be used to help evaluate for increased intracranial pressure. The first tool is measurement of the diameter of the optic nerve sheath, assessed at $3 \mathrm{~mm}$ deep to the optic disc. When measuring this, it is important that the optic nerve is parallel to the ultrasound beam. Clinicians should consider measuring in two planes if there is concern regarding inaccurate measurement. The size threshold varies between studies. One systematic review using $5 \mathrm{~mm}$ for adults, $4.5 \mathrm{~mm}$ for children (ages $1-17$ years), and $4 \mathrm{~mm}$ for infants (age $<1$ year) reported $96 \%$ sensitivity and $92 \%$ specificity [10]. However, a more recent review found that despite relatively high sensitivity and specificity, the thresholds varied significantly across studies, with the optimal cutoff ranging from 4.8 to $6.4 \mathrm{~mm}$ [11]. Lower threshold values increase the sensitivity, while high values increase the specificity [11]. While further studies are needed, sonographers should consider selecting the value threshold based upon whether they are seeking to be more sensitive or specific in their diagnosis of elevated increased intracranial pressure. Another technique that can be utilized to assess for increased intracranial pressure is sonographic papilledema (also known as optic disc elevation [ODE]), which assesses for elevation of the optic disc above the level of the retina. While the literature is more limited, one study reported that an ODE $>0.6 \mathrm{~mm}$ was $82 \%$ sensitive and $76 \%$ specific, while an ODE $>1.0 \mathrm{~mm}$ was $73 \%$ sensitive and $100 \%$ specific [12].

\section{Case resolution}

The clinician utilizes POCUS and identifies a retinal detachment with intact macula. The clinician notifies ophthalmology who is able to intervene before the retinal detachment has progressed further.

Funding None.

\section{Declarations}

Conflict of interest The authors declare that they have no conflict of interest.

\section{References}

1. Miller DL, Abo A, Abramowicz JS, et al. Diagnostic ultrasound safety review for point-of-care ultrasound practitioners. J Ultrasound Med. 2020;39(6):1069-84.

2. Khattab E, Hoffmann B, Schafer J, et al. Examiner position in ocular point-of-care ultrasound: a proposed technique. J Emerg Med. 2019;56(6):684-6.

3. Tandon A, Khullar T, Bhatt S. Sonography in acute ocular pathology: a kaleidoscopic view. Emerg Radiol. 2019;26(2):241-8.

4. Hollands H, Johnson D, Brox AC, Almeida D, Simel DL, Sharma S. Acute-onset floaters and flashes: is this patient at risk for retinal detachment? JAMA. 2009;302(20):2243-9.

5. Gottlieb M, Holladay D, Peksa GD. Point-of-care ocular ultrasound for the diagnosis of retinal detachment: a systematic review and meta-analysis. Acad Emerg Med. 2019;26(8):931-9.

6. Propst SL, Kirschner JM, Strachan CC, Roumpf SK, Menard LM, Sarmiento EJ, Hunter BR. Ocular point-of-care ultrasonography to diagnose posterior chamber abnormalities: a systematic review and meta-analysis. JAMA Netw Open. 2020;3(2):e1921460.

7. Lahham S, Shniter I, Thompson M, et al. Point-of-care ultrasonography in the diagnosis of retinal detachment, vitreous hemorrhage, and vitreous detachment in the emergency department. JAMA Netw Open. 2019;2(4):e192162.

8. Baker N, Amini R, Situ-LaCasse EH, et al. Can emergency physicians accurately distinguish retinal detachment from posterior vitreous detachment with point-of-care ocular ultrasound? Am J Emerg Med. 2018;36(5):774-6.

9. Woo MY, Hecht N, Hurley B, Stitt D, Thiruganasambandamoorthy $\mathrm{V}$. Test characteristics of point-of-care ultrasonography for the diagnosis of acute posterior ocular pathology. Can J Ophthalmol. 2016;51(5):336-41.

10. Ohle R, McIsaac SM, Woo MY, Perry JJ. Sonography of the optic nerve sheath diameter for detection of raised intracranial pressure compared to computed tomography: a systematic review and meta-analysis. J Ultrasound Med. 2015;34(7):1285-94.

11. Fernando SM, Tran A, Cheng W, et al. Diagnosis of elevated intracranial pressure in critically ill adults: systematic review and meta-analysis. BMJ. 2019;366:14225.

12. Teismann N, Lenaghan P, Nolan R, Stein J, Green A. Point-of-care ocular ultrasound to detect optic disc swelling. Acad Emerg Med. 2013;20(9):920-5. 